\title{
Who should avoid single incision laparoscopic cholecystectomy for benign gallbladder disease: Lesson learned from 1,405 consecutive patients in a single center
}

\author{
Seung Jae LEE, In Seok CHOI*, Ju Ik MOON
}

DDepartment of Surgery, Konyang University College of Medicine, Daejeon, Korea

Introduction: The purpose of this study is to recommend an optimal indication of single incision laparoscopic cholecystectomy (SILC). Methods: We retrospectively reviewed the 1,405 consecutive patients who underwent SILC at a single institution between April 2010 and June 2020. We defined difficult surgery (DS; conversion to multiport or open, adjacent organ injury, operation time $\geq 90$ minutes, or estimated blood loss $\geq 100$ milliliters) and poor postoperative outcomes (PPO; postoperative hospital stays $\geq 5$ days, or postoperative complication $\geq$ grade II Clavien-Dindo classification). Subgroup analysis of acute cholecystitis (AC) was conducted according to the Tokyo guideline 18 .

Results: Of the 1,405 patients, 338 were for gallbladder (GB) stone, 121 were for GB polyp, 478 were for chronic cholecystitis, and 423 were for AC. The conversion and postoperative complication rate were $2.4 \%$ and $3.5 \%$, the mean operation time and length of postoperative hospital stay were 51.8 minutes and 2.5 days. 89 and 94 patients were included in DS group and PPO group, respectively. In multivariable analysis, both grade I or grade II/III AC, and body mass index $\geq 30 \mathrm{~kg} / \mathrm{m}^{2}$ were statistically significant predictors of DS. Grade II/III AC and age $\geq 70$ years were statistically significant predictors of PPO. In subgroup analysis of AC, grade II/III AC group had longer operation time (57.2 vs. 67.4 minutes, $p<0.001)$, postoperative hospital stays ( 2.7 vs. 3.7 days, $p=0.001)$, higher complication rate $(4.2$ vs. $15.8 \%, p<0.001)$, and conversion rate $(3.9$ vs. $15.8 \%, p<0.001)$ than grade I AC group.

Conclusions: SILC should be avoided in patients with grade II/III AC for better surgical outcomes. 